\title{
On an Operator Preserving Inequalities between Polynomials
}

\author{
Nisar Ahmad Rather, Mushtaq Ahmad Shah, Mohd. Ibrahim Mir \\ Post-Graduate Department of Mathematics, Kashmir University, Srinagar, India \\ Email: \{dr.narather, mushtaqa022\}@gmail.com
}

Received April 11, 2012; revised May 8, 2012; accepted May 16, 2012

\section{ABSTRACT}

Let $P_{n}$ be the class of polynomials $P(z)$ of degree $n$ and $B_{n}$ a family of operators that map $P_{n}$ into itself. For $B \in B_{n}$, we investigate the dependence of

$$
\left|B[P(R z)]-\alpha B[P(r z)]+\beta\left\{\left(\frac{R+1}{r+1}\right)^{n}-|\alpha|\right\} B[P(r z)]\right|
$$

on the maximum modulus of $P(z)$ on $|z|=1$ for arbitrary real or complex numbers $\alpha, \beta$ with $|\alpha| \leq 1,|\beta| \leq 1$ and $R>r \geq 1$, and present certain sharp operator preserving inequalities between polynomials.

Keywords: Component Polynomials; B-Operator; Complex Domain

\section{Introduction to the Statement of Results}

Let $P_{n}(z)$ denote the space of all complex polynomials $P(z)=\sum_{j=0}^{n} a_{j} z^{j}$ of degree $n$. If $P \in P_{n}$, then concerning the estimate of the maximum of $\left|P^{\prime}(z)\right|$ on the unit circle $|z|=1$ and the estimate of the maximum of $|P(z)|$ on a larger circle $|z|=R>1$, we have

$$
\max _{|z|=1}\left|P^{\prime}(z)\right| \leq n \max _{|z|=1}|P(z)|
$$

and

$$
\max _{|z|=R>1}|P(z)| \leq R^{n} \max _{|z|=1}|P(z)| .
$$

Inequality (1) is an immediate consequence of S. Bernstein's theorem (see [1-3]) on the derivative of a trigonometric polynomial. Inequality (2) is a simple deduction from the maximum modulus principle (see [4, p. 346] or [5, p. 158]). If we restrict ourselves to the class of polynomials $P \in P_{n}$ having no zero in $|z|<1$, then (1) and (2) can be replaced by

$$
\max _{|z|=1}\left|P^{\prime}(z)\right| \leq \frac{n}{2} \max _{|z|=1}|P(z)|
$$

and

$$
\max _{|z|=R>1}|P(z)| \leq \frac{R^{n}+1}{2} \max _{|z|=1}|P(z)| .
$$

Inequality (3) was conjectured by Erdös and later verified by Lax [6]. Ankeny and Rivlin [7] used Inequality (3) to prove Inequality (4).

As a compact generalization of Inequalities (1) and (2), Aziz and Rather [8] have shown that if $P \in P_{n}$, then for every real or complex number $\alpha$ with $|\alpha| \leq 1, R>1$ and $|z|=1$,

$$
|P(R z)-\alpha P(z)| \leq\left|R^{n}-\alpha\right||z|^{n} \max _{|z|=1}|P(z)| .
$$

The result is sharp.

As a corresponding compact generalization of Inequalities (3) and (4), they [8] have also shown that if $P \in P_{n}$, and $P(z) \neq 0$ for $|z|<1$, then for every real or complex number $\alpha$ with $|\alpha| \leq 1, R \geq 1$,

$$
\begin{aligned}
& |P(R z)-\alpha P(z)| \\
& \leq \frac{1}{2}\left\{\left|R^{n}-\alpha\right||z|^{n}+|1-\alpha|\right\} \max _{|z|=1}|P(z)|
\end{aligned}
$$

for $|z| \geq 1$. The result is sharp and equality in (6) holds for $P(z)=a z^{n}+b,|a|=|b|=1$.

Consider an operator $B$ which carries a polynomial $P \in P_{n}$ into

$$
\begin{aligned}
B[P(z)]= & \lambda_{0} P(z)+\lambda_{1}\left(\frac{n z}{2}\right) \frac{P^{\prime}(z)}{1 !} \\
& +\lambda_{2}\left(\frac{n z}{2}\right)^{2} \frac{P^{\prime \prime}(z)}{2 !},
\end{aligned}
$$

where $\lambda_{0}, \lambda_{1}$ and $\lambda_{2}$ are such that all the zeros of 


$$
u(z)=\lambda_{0}+\lambda_{1} C(n, 1) z+\lambda_{2} C(n, 2) z^{2}
$$

lie in the half plane

$$
|z| \leq|z-n / 2|
$$

As a generalization of the Inequalities (1) and (2), Q.I. Rahman [9] proved that if $P \in P_{n}$, then for $|z| \geq 1$,

$$
|B[P(z)]| \leq\left|B\left[z^{n}\right]\right| \max _{|z|=1}|P(z)| .
$$

and if $P(z) \neq 0$ for $|z|<1$, then for $|z| \geq 1$,

$$
|B[P(z)]| \leq \frac{1}{2}\left\{\left|B\left[z^{n}\right]\right|+\left|\lambda_{0}\right|\right\} \max _{|z|=1}|P(z)| .
$$

(see [9], Inequality (5.2) and (5.3)).

In this paper, we consider a problem of investigating the dependence of

$$
\left|B[P(R z)]-\alpha B[P(r z)]+\beta\left\{\left(\frac{R+1}{r+1}\right)^{n}-|\alpha|\right\} B[P(r z)]\right|
$$

on the maximum modulus of $P(z)$ on $|z|=1$ for arbitrary real or complex numbers $\alpha, \beta$ with $|\alpha| \leq 1,|\beta| \leq 1$ and $R>r \geq 1$, and develop a unified method for arriving at these results. In this direction we first present the following interesting result which is compact generalization of the Inequalities (1), (2), (5) and (10).

Theorem 1. If $P \in P_{n}$, then for arbitrary real or complex numbers $\alpha$ and $\beta$ with $|\alpha| \leq 1,|\beta| \leq 1 \quad R>r \geq 1$ and $|z| \geq 1$,

$$
\begin{aligned}
& |B[P(R z)]+\phi(R, r, \alpha, \beta) B[P(r z)]| \\
& \leq\left|R^{n}+\phi(R, r, \alpha, \beta) r^{n}\right|\left|B\left[z^{n}\right]\right| \max _{|z|=1}|P(z)|
\end{aligned}
$$

where

$$
\phi(R, r, \alpha, \beta)=\beta\left\{\left(\frac{R+1}{r+1}\right)^{n}-|\alpha|\right\}-\alpha .
$$

The result is best possible and equality in (12) holds for $P(z)=\lambda z^{n} ; \lambda \neq 0$.

Remark 1. For $\beta=0$, from Inequality (12), we have for $P \in P_{n},|\alpha| \leq 1,|z| \geq 1$ and $R>r \geq 1$,

$$
\begin{aligned}
& |B[P(R z)]-\alpha B[P(r z)]| \\
& \leq\left|R^{n}-\alpha r^{n}\right|\left|B\left[z^{n}\right]\right| \max _{|z|=1}|P(z)| .
\end{aligned}
$$

Remark 2. For $\alpha=0$ and $\beta=0$, Inequality (12) reduces to

$$
\begin{aligned}
|B[P(R z)]| & \leq R^{n}\left|B\left[z^{n}\right]\right| \max _{|z|=1}|P(z)| \\
& =\left|B\left[R^{n} z^{n}\right]\right| \max _{|z|=1}|P(z)| .
\end{aligned}
$$

for $P \in P_{n},|z| \geq 1$ and $R>1$, which contains Inequality (10) as a special case.

Remark 3. For $\alpha=0$, Inequality (12) yields,

$$
\begin{aligned}
& \left|B[P(R z)]+\beta\left(\frac{R+1}{r+1}\right)^{n} B[P(r z)]\right| \\
& \leq\left|R^{n}+\beta\left(\frac{R+1}{r+1}\right) r^{n}\right|\left|B\left[z^{n}\right]\right| \max _{|z|=1}|P(z)|
\end{aligned}
$$

for $|z| \geq 1, R>r \geq 1$ and $|\beta| \leq 1$.

If we choose $\lambda_{0}=\lambda_{2}=0$ in (12) and noting that all the zeros of $u(z)$ defined by (8) lie in the half plane (9), we get:

Corollary 1. If $P \in P_{n}$, then for all real or complex numbers $\alpha$ and $\beta$ with $|\alpha| \leq 1,|\beta| \leq 1, R>r \geq 1$ and $|z| \geq 1$,

$$
\begin{aligned}
& \left|R P^{\prime}(R z)+\phi(R, r, \alpha, \beta) r P^{\prime}(r z)\right| \\
& \leq n\left|R^{n}+\phi(R, r, \alpha, \beta) r^{n}\right||z|^{n-1} \max _{|z|=1}|P(z)| .
\end{aligned}
$$

where $\phi(R, r, \alpha, \beta)$ is defined as in Theorem 1. The result is sharp and equality in (16) holds for $P(z)=\lambda z^{n}$, $\lambda \neq 0$.

For the case $B[P(z)]=P(z)$, from (12) we obatin for all real or complex numbers $\alpha$ and $\beta$ with $|\alpha| \leq 1$, $|\beta| \leq 1, \quad R>r \geq 1$ and $|z| \geq 1$,

$$
\begin{aligned}
& |P(R z)+\phi(R, r, \alpha, \beta) P(r z)| \\
& \leq\left|R^{n}+\phi(R, r, \alpha, \beta) r^{n}\right||z|^{n} \max _{|z|=1}|P(z)| .
\end{aligned}
$$

Inequality (17) is equivalent to the Inequality (5) for $|z| \geq 1$ and $\beta=0$. For $\alpha=0$ and $\beta=0$, Inequality (17) includes Inequality (2) as a special case.

Next we use Theorem 1 to prove the following result.

Theorem 2. If $P \in P_{n}$, then for arbitrary real or complex numbers $\alpha$ and $\beta$ with $|\alpha| \leq 1,|\beta| \leq 1, R>r \geq 1$ and $|z| \geq 1$,

$$
\begin{aligned}
|B[P(R z)]+\phi(R, r, \alpha, \beta) B[P(r z)]| \\
\quad+|B[Q(R z)]+\phi(R, r, \alpha, \beta) B[Q(r z)]| \\
\leq\left\{\left|R^{n}+\phi(R, r, \alpha, \beta) r^{n}\right|\left|B\left[z^{n}\right]\right|\right. \\
\left.\quad+|1+\phi(R, r, \alpha, \beta)|\left|\lambda_{0}\right|\right\} \max _{|z|=1}|P(z)|
\end{aligned}
$$

where $Q(z)=z^{n} \overline{P(1 / \bar{z})}$ and $\phi(R, r, \alpha, \beta)$ is defined as in Theorem 1 .

The result is sharp and equality in (18) holds for $P(z)=\lambda z^{n}, \lambda \neq 0$.

Remark 4. Theorem 2 includes some well known polynomial inequalities as special cases. For example, inequality (18) reduces to a result due to Q. I. Rahman ([8], Inequality (5.2) with $\alpha=0$ and $\beta=0$ ). Also for 
$\beta=0$, Inequality (18) gives

$$
\begin{aligned}
& |B[P(R z)]-\alpha B[P(r z)]|+|B[Q(R z)]-\alpha B[Q(r z)]| \\
& \leq\left\{\left|R^{n}-\alpha r^{n}\right|\left|B\left[z^{n}\right]\right|+|1-\alpha|\left|\lambda_{0}\right|\right\} \max _{|z|=1}|P(z)|,
\end{aligned}
$$

for $|\alpha| \leq 1, \quad R>r \geq 1$ and $|z| \geq 1$.

If we take $\lambda_{0}=\lambda_{2}=0$ in (18), we get:

Corollary 2. If $P \in P_{n}$, then for all real or complex numbers $\alpha$ and $\beta$ with $|\alpha| \leq 1,|\beta| \leq 1, \quad R>r \geq 1$ and $|z| \geq 1$,

$$
\begin{aligned}
& \left|R P^{\prime}(R z)+\phi(R, r, \alpha, \beta) r P^{\prime}(r z)\right| \\
& \quad+\left|R Q^{\prime}(R z)+\phi(R, r, \alpha, \beta) r Q^{\prime}(r z)\right| \\
& \leq n\left|R^{n}+\phi(R, r, \alpha, \beta) r^{n}\right||z|^{n-1} \max _{|z|=1}|P(z)| .
\end{aligned}
$$

where $\phi(R, r, \alpha, \beta)$ is defined as in Theorem 1. The result is sharp and equality in (20) holds for $P(z)=\lambda z^{n}$, $\lambda \neq 0$.

For $\lambda_{1}=\lambda_{2}=0$ and $\alpha=1, \beta=0$, Theorem 2 includes a result due to A. Aziz and Rather [2] as a special case.

Inequality (12) can be sharpened if we restrict ourselves to the class of polynomials $P \in P_{n}$ having no zeros in $|z|<1$. In this direction we next prove the following result which is a compact generalization of the Inequalities (3), (4) and (6).

Theorem 3. If $P \in P_{n}$ and $P(z) \neq 0$ for $|z|<1$, then for arbitrary real or complex numbers $\alpha$ and $\beta$ with $|\alpha| \leq 1,|\beta| \leq 1, \quad R>r \geq 1$ and $|z| \geq 1$,

$$
\begin{aligned}
& |B[P(R z)]+\phi(R, r, \alpha, \beta) B[P(r z)]| \\
& \leq \frac{1}{2}\left\{\left|R^{n}+\varphi(R, r, \alpha, \beta) r^{n}\right|\left|B\left[z^{n}\right]\right|\right. \\
& \left.\quad+|1+\varphi(R, r, \alpha, \beta)|\left|\lambda_{0}\right|\right\} \max _{|z|=1}|P(z)|
\end{aligned}
$$

where $\phi(R, r, \alpha, \beta)$ is defined as in Theorem 1. The result is sharp and equality in (21) holds for $P(z)=z^{n}+1$.

Remark 5. Inequality (11) is a special case of the Inequality (21) for $\alpha=0$ and $\beta=0$. If we choose $\lambda_{0}=$ $\lambda_{2}=0$ in (21) and note that all the zeros of $u(z)$ defined by (8) lie in the half plane defined by (9), it follows that if $P \in P_{n}$ and $P(z) \neq 0$ for $|z|<1$, then for $|z| \geq 1$, $R>r \geq 1$ and $|\alpha| \leq 1,|\beta| \leq 1$,

$$
\begin{aligned}
& \left|R P^{\prime}(R z)+\phi(R, r, \alpha, \beta) r P^{\prime}(r z)\right| \\
& \leq \frac{n}{2}\left|R^{n}+\phi(R, r, \alpha, \beta) r^{n}\right||z|^{n-1} \max _{|z|=1}|P(z)| .
\end{aligned}
$$

Setting $\alpha=0$ in (22), we obtain for $P \in P_{n}$,

$$
\begin{aligned}
& \left|R P^{\prime}(R z)+\beta\left(\frac{R+1}{r+1}\right)^{n} r P^{\prime}(r z)\right| \\
& \leq \frac{n}{2}\left|R^{n}+\beta\left(\frac{R+1}{r+1}\right)^{n} r^{n}\right||z|^{n-1} \max _{|z|=1}|P(z)|
\end{aligned}
$$

for $|z| \geq 1, \quad R>r \geq 1$ and $|\beta| \leq 1$.

Taking $\alpha=\beta=0$ in (22), we obtain for $P \in P_{n}$, $|z| \geq 1$ and $R>1$,

$$
\left|P^{\prime}(R z)\right| \leq \frac{n}{2} R^{n-1}|z|^{n-1} \max _{|z|=1}|P(z)|,
$$

which in particular gives Inequality (3).

Next choosing $\lambda_{1}=\lambda_{2}=0$ in (21), we immediately get for $|z| \geq 1, \quad R>r \geq 1$ and $|\alpha| \leq 1,|\beta| \leq 1$,

$$
\begin{aligned}
& |P(R z)+\phi(R, r, \alpha, \beta) P(r z)| \\
& \leq \frac{1}{2}\left\{\left|R^{n}+\phi(R, r, \alpha, \beta) r^{n}\right||z|^{n}\right. \\
& \quad+|1+\phi(R, r, \alpha, \beta)|\} \max _{|z|=1}|P(z)|,
\end{aligned}
$$

which is a compact generalization of the Inequalities (3), (4) and (6). The result is sharp and equality in (25) holds for $P(z)=a z^{n}+b,|a|=|b|=1$.

If we put $\beta=0$ in (25), we get the following result.

Corollary 3. If $P \in P_{n}$, and $P(z) \neq 0$ for $|z|<1$, then for every real or complex number $\alpha$ with $|\alpha| \leq 1$, $R>r \geq 1$ and $|z| \geq 1$,

$$
\begin{aligned}
& |P(R z)-\alpha P(r z)| \\
& \leq \frac{1}{2}\left\{\left|R^{n}-\alpha r^{n}\right||z|^{n}+|1-\alpha|\right\} \max _{|z|=1}|P(z)| .
\end{aligned}
$$

A polynomial $P \in P_{n}$ is said to be self-inversive if $P(z)=Q(z)$ where $Q(z)=z^{n} \overline{P(1 / \bar{z})}$. It is known $[6$, 10] that if $P \in P_{n}$ is a self-inversive polynomial, then

$$
\max _{|z|=1}\left|P^{\prime}(z)\right| \leq \frac{n}{2} \max _{|z|=1}|P(z)| .
$$

Here finally, we establish the following result for self-inversive polynomials

Theorem 4. If $P \in P_{n}$ is a self-inversive polynomial, then for arbitrary real or complex numbers $\alpha$ and $\beta$ with $|\alpha| \leq 1,|\beta| \leq 1, \quad R>r \geq 1$ and $|z| \geq 1$,

$$
\begin{aligned}
& |B[P(R z)]+\phi(R, r, \alpha, \beta) B[P(r z)]| \\
& \leq \frac{1}{2}\left\{\left|R^{n}+\phi(R, r, \alpha, \beta) r^{n}\right|\left|B\left[z^{n}\right]\right|\right. \\
& \left.\quad+|1+\phi(R, r, \alpha, \beta)|\left|\lambda_{0}\right|\right\} \max _{|z|=1}|P(z)|
\end{aligned}
$$

where $\phi(R, r, \alpha, \beta)$ is defined as in Theorem 1. The result is sharp and equality in (21) holds for $P(z)=z^{n}+1$.

The following result is an immediate consequence of 
Theorem 4.

Corollary 4. If $P \in P_{n}$ is a self-inversive polynomial, then for arbitrary real or complex numbers $\alpha$ and $\beta$ with $|\alpha| \leq 1,|\beta| \leq 1, R>r \geq 1$ and $|z| \geq 1$,

$$
\begin{aligned}
& |P(R z)+\phi(R, r, \alpha, \beta) P(r z)| \\
& \leq \frac{1}{2}\left\{\left|R^{n}+\phi(R, r, \alpha, \beta) r^{n}\right||z|^{n}\right. \\
& \quad+|1+\phi(R, r, \alpha, \beta)|\} \max _{|z|=1}|P(z)| .
\end{aligned}
$$

where $\phi(R, r, \alpha, \beta)$ is defined as in Theorem 1. The result is best possible

For $\beta=0$ the Inequality (29) reduces to

$$
\begin{aligned}
& |P(R z)-\alpha P(r z)| \\
& \leq \frac{1}{2}\left\{\left|R^{n}-\alpha r^{n}\right||z|^{n}+|1-\alpha|\right\} \max _{|z|=1}|P(z)| .
\end{aligned}
$$

Remark 6. Inequality (6) is a special case of the Inequality (30). Many other interesting results can be deduced from Theorem 4 in the same way as we have deduced from Theorem 1 and Theorem.

\section{Lemmas}

For the proofs of these theorems, we need the following lemmas. The first lemma can be easily proved.

Lemma 1. If $P \in P_{n}$ and $P(z)$ has all its zeros in $|z| \leq 1$, then for every $R>r \geq 1$ and $|z|=1$,

$$
|P(R z)| \geq\left(\frac{R+1}{r+1}\right)^{n}|P(r z)| .
$$

The next Lemma follows from corollary 18.3 of [11, p. 65].

Lemma 2. If $P \in P_{n}$ and $P(z)$ has all its zeros in $|z| \leq 1$, then all the zeros of $B[P(z)]$ also lie in $z \mid \leq 1$.

Lemma 3. If $P \in P_{n}$ and $P(z)$ does not vanish in $|z|<1$, then for arbitrary real or complex numbers $\alpha$ and $\beta$ with $|\alpha| \leq 1,|\beta| \leq 1, R>r \geq 1$ and $|z|=1$,

$$
\begin{aligned}
& |B[P(R z)]+\phi(R, r, \alpha, \beta) B[P(r z)]| \\
& \leq|B[Q(R z)]+\phi(R, r, \alpha, \beta) B[Q(r z)]|
\end{aligned}
$$

where $Q(z)=z^{n} \overline{P(1 / \bar{z})}$ and $\phi(R, r, \alpha, \beta)$ is defined as in Theorem 1.

The result is sharp and equality in (32) holds for $P(z)=z^{n}+1$.

Proof of Lemma 3. Since the polynomial $P(z)$ has all its zeros in $|z| \geq 1$ for every real or complex number $\gamma$ with $|\gamma|>1$, the polynomial $g(z)=P(z)-\gamma Q(z)$, where $Q(z)=z^{n} \overline{P(1 / \bar{z})}$, has all its zeros in $|z| \geq 1$ with atleast one zero in $|z|<1$, so that we can write

$$
g(z)=\left(z-t e^{i \delta}\right) h(z),
$$

where $t<1$ and $h(z)$ is a polynomial of degree $n-1$ having all its zeros in $|z| \leq 1$.

Applying lemma 1 to the polynomial $h(z)$, we obtain for $R>r \geq 1$ and $0 \leq \theta<2 \pi$,

$$
\begin{aligned}
& \left|g\left(\operatorname{Re}^{i \theta}\right)\right|=\left|\operatorname{Re}^{i \theta}-t e^{i \delta}\right|\left|h\left(\operatorname{Re}^{i \theta}\right)\right| \\
& \geq\left|\operatorname{Re}^{i \theta}-t e^{i \delta}\right|\left(\frac{R+1}{r+1}\right)^{n-1}\left|h\left(r e^{i \theta}\right)\right|
\end{aligned}
$$

This implies for $R>r \geq 1$ and $0 \leq \theta<2 \pi$,

$$
\left(\frac{r+t}{R+t}\right)\left|g\left(\operatorname{Re}^{i \theta}\right)\right| \geq\left(\frac{R+1}{r+1}\right)^{n-1}\left|g\left(r e^{i \theta}\right)\right| .
$$

Since $R>r \geq 1>t$ so that $g\left(\operatorname{Re}^{i \theta}\right) \neq 0$ for $0 \leq \theta<2 \pi$ and $\frac{1+r}{1+R}>\frac{r+t}{R+t}$, from Inequality (33), we obtain for $R>r \geq 1$ and $0 \leq \theta<2 \pi$,

$$
\left|g\left(\operatorname{Re}^{i \theta}\right)\right|>\left(\frac{R+1}{r+1}\right)^{n}\left|g\left(r e^{i \theta}\right)\right| .
$$

Equivalently,

$$
|g(R z)|>\left(\frac{R+1}{r+1}\right)^{n}|g(r z)|
$$

for $|z|=1$ and $R>r \geq 1$. Hence for every real or complex number $\alpha$ with $|\alpha| \leq 1$ and $R>r \geq 1$ we have

$$
\begin{aligned}
|g(R z)-\alpha g(r z)| & \geq|g(R z)|-|\alpha||g(r z)| \\
& >\left\{\left(\frac{R+1}{r+1}\right)^{n}-|\alpha|\right\}|g(r z)|
\end{aligned}
$$

for $|z|=1$. Also, Inequality (34) can be written as

$$
\left|g\left(r e^{i \theta}\right)\right|<\left(\frac{r+1}{R+1}\right)^{n}\left|g\left(\operatorname{Re}^{i \theta}\right)\right|
$$

for every $R>r \geq 1$ and $0 \leq \theta<2 \pi$ Since

$g\left(\operatorname{Re}^{i \theta}\right) \neq 0$ and $\left(\frac{r+1}{R+1}\right)^{n}<1$, from inequality (36), we obtain for $0 \leq \theta<2 \pi$ and $R>r \geq 1$,

$$
\left|g\left(r e^{i \theta}\right)\right|<\left|g\left(\operatorname{Re}^{i \theta}\right)\right| \text {. }
$$

Equivalently,

$$
|g(r z)|<|g(R z)| \text { for }|z|=1 .
$$

Since all the zeros of $g(R z)$ lie in $|z| \leq(1 / R)<1$, a direct application of Rouche's theorem shows that the polynomial $g(R z)-\alpha g(r z)$ has all its zeros in $|z|<1$ for every real or complex number $\alpha$ with $|\alpha| \leq 1$. Applying Rouche's theorem again, it follows from (35) that for arbitrary real or complex numbers $\alpha, \beta$ with $|\alpha| \leq 1$, 
$|\beta| \leq 1$ and $R>r \geq 1$, all the zeros of the polynomial

$$
\begin{aligned}
F(z)= & g(R z)+\varphi(R, r, \alpha, \beta) g(r z) \\
= & {[\{P(R z)-\gamma Q(R z)\}} \\
& +\phi(R, r, \alpha, \beta)\{P(R z)-\gamma Q(R z)\}] \\
= & {[P(R z)+\phi(R, r, \alpha, \beta) P(r z)] } \\
& -\gamma[Q(R z)+\phi(R, r, \alpha, \beta) Q(r z)]
\end{aligned}
$$

lie in $|z|<1$ with $|\gamma| \geq 1$. Applying Lemma 2 to the polynomial $F(z)$ and noting that $B$ is a linear operator, it follows that all the zeros of the polynomial

$$
\begin{aligned}
T(z)= & B[F(z)] \\
= & \{B[P(R z)]+\phi(R, r, \alpha, \beta) B[P(r z)]\} \\
& -\gamma\{B[Q(R z)]+\phi(R, r, \alpha, \beta) B[Q(r z)]\}
\end{aligned}
$$

lie in $|z|<1$ for all real or complex numbers $\alpha, \beta, \gamma$ with $|\alpha| \leq 1,|\beta| \leq 1,|\gamma| \leq 1$ and $R>r \geq 1$. This implies

$$
\begin{aligned}
& |B[P(R z)]+\phi(R, r, \alpha, \beta) B[P(r z)]| \\
& \leq|B[Q(R z)]+\phi(R, r, \alpha, \beta) B[Q(r z)]|
\end{aligned}
$$

for $|z| \geq 1,|\alpha| \leq 1,|\beta| \leq 1$ and $R>r \geq 1$. If Inequality (38) is not true, then there is a point $z=w$ with $|w| \geq 1$ such that

$$
\begin{aligned}
& \left|\{B[P(R z)]+\phi(R, r, \alpha, \beta) B[P(r z)]\}_{z=w}\right| \\
& >\left|\{B[Q(R z)]+\phi(R, r, \alpha, \beta) B[Q(r z)]\}_{z=w}\right| .
\end{aligned}
$$

But all the zeros of $Q(z)$ lie in $|z| \leq 1$, therefore, it follows (as in case of $g(z)$ ) that all the zeros of

$$
Q(R z)+\phi(R, r, \alpha, \beta) Q(r z)
$$

lie in $|z|<1$. Hence by Lemma 2, all the zeros of

$$
B[Q(R z)]+\phi(R, r, \alpha, \beta) B[Q(r z)]
$$

lie in $|z|<1$, so that

$$
\{B[Q(R z)]+\phi(R, r, \alpha, \beta) B[Q(r z)]\}_{z=w} \neq 0 .
$$

We take

$$
\gamma=\frac{\{B[P(R z)]+\phi(R, r, \alpha, \beta) B[P(r z)]\}_{z=w}}{\{B[Q(R z)]+\phi(R, r, \alpha, \beta) B[Q(r z)]\}_{z=w}},
$$

then $\gamma$ is a well defined real or complex number with $|\gamma|>1$ and with this choice of $\gamma$, from (37) we obtain $T(w)=0$ where $|w| \geq 1$. This contradicts the fact that all the zeros of $T(z)$ lie in $|z|<1$. Thus

$$
\begin{aligned}
& |B[P(R z)]+\phi(R, r, \alpha, \beta) B[P(r z)]| \\
& \leq|B[Q(R z)]+\phi(R, r, \alpha, \beta) B[Q(r z)]|
\end{aligned}
$$

for $|z| \geq 1,|\alpha| \leq 1,|\beta| \leq 1$ and $R>r \geq 1$. This proves (38) and hence Lemma 3.

\section{Proofs of the Theorems}

Proof of Theorem 1. Let $M=\max _{|z|=1}|P(z)|$, then $|P(z)| \leq M$ for $|z|=1$. By Rouche's Theorem, it follows that all the zeros of the polynomial $H(z)=P(z)-\lambda M z^{n}$ lie in $|z|<1$ for every real or complex number $\lambda$ with $|\lambda|>1$, therefore, as before (as in Lemma 3), we conclude that all the zeros of the polynomial

$$
G(z)=H(R z)+\phi(R, r, \alpha, \beta) H(r z)
$$

lie in $|z|<1$ for all real or complex numbers $\alpha$ and $\beta$ with $|\alpha| \leq 1$ and $|\beta| \leq 1$. Hence by Lemma 2 , the polynomial

$$
\begin{aligned}
T(z)= & B[G(z)] \\
= & B[H(R z)]+\phi(R, r, \alpha, \beta) B[H(r z)] \\
= & B[P(R z)]+\phi(R, r, \alpha, \beta) B[P(r z)] \\
& +\lambda\left[R^{n}+\phi(R, r, \alpha, \beta) r^{n}\right] B\left[z^{n}\right] M
\end{aligned}
$$

has all its zeros in $|z|<1$ for every real or complex number $\lambda$ with $|\lambda|>1$. This implies for every real or complex numbers $\alpha$ and $\beta$ with $|\alpha| \leq 1,|\beta| \leq 1$ and $R>r \geq 1$,

$$
\begin{aligned}
& |B[P(R z)]+\phi(R, r, \alpha, \beta) B[P(r z)]| \\
& \leq\left|R^{n}+\phi(R, r, \alpha, \beta) r^{n}\right|\left|B\left[z^{n}\right]\right| M \text { for }|z| \geq 1
\end{aligned}
$$

If Inequality (40) is not true, then there is a point $z=w$ with $|w| \geq 1$ such that

$$
\begin{aligned}
& \left|\{B[P(R z)]+\phi(R, r, \alpha, \beta) B[P(r z)]\}_{z=w}\right| \\
& >\left\{\left|R^{n}+\phi(R, r, \alpha, \beta) r^{n}\right|\left|B\left[z^{n}\right]\right|_{z=w} M\right\} .
\end{aligned}
$$

Since $\left\{B\left[z^{n}\right]\right\}_{z=w} \neq 0$, we take

$$
\lambda=\frac{\{B[P(R z)]+\phi(R, r, \alpha, \beta) B[P(r z)]\}_{z=w}}{\left[R^{n}+\phi(R, r, \alpha, \beta) r^{n}\right]\left\{B\left[z^{n}\right]\right\}_{z=w} M},
$$

so that $\lambda$ is a well defined real or complex number with $|\lambda|>1$ and with this choice of $\lambda$, from (39) we get $T(w)=0$ where $|w| \geq 1$. This contradicts the fact that all the zeros of $T(z)$ lie in $|z|<1$. Thus for every real or complex numbers $\alpha$ and $\beta$ with $|\alpha| \leq 1,|\beta| \leq 1$ and $R>r \geq 1$, 


$$
\begin{aligned}
& |B[P(R z)]+\phi(R, r, \alpha, \beta) B[P(r z)]| \\
& \leq\left|R^{n}+\phi(R, r, \alpha, \beta) r^{n}\right|\left|B\left[z^{n}\right]\right| M \text { for }|z| \geq 1 .
\end{aligned}
$$

This completes the proof of Theorem 1 .

Proof of Theorem 2. Let $M=\max _{|z|=1}|P(z)|$, then $|P(z)| \leq M$ for $|z|=1$. If $\mu$ is any real or complex number with $|\mu|>1$, then by Rouche's Theorem, the polynomial $f(z)-\mu M$ does not vanish in $|z|<1$. Applying Lemma 3 to the polynomial $f(z)$ and using the fact that $B$ is a linear operator, it follows that for all real or complex numbers $\alpha$ and $\beta$ with $|\alpha| \leq 1,|\beta| \leq 1$, $R>r \geq 1$ and for $|z| \geq 1$

$$
\begin{aligned}
& |B[f(R z)]+\phi(R, r, \alpha, \beta) B[f(r z)]| \\
& \leq\left|B\left[f^{*}(R z)\right]+\phi(R, r, \alpha, \beta) B\left[f^{*}(r z)\right]\right|
\end{aligned}
$$

where

$$
\begin{aligned}
f^{*}(z) & =z^{n} \overline{f(1 / \bar{z})}=z^{n} \overline{P(1 / \bar{z})}-\bar{\mu} M z^{n} \\
& =Q(z)-\bar{\mu} M z^{n},
\end{aligned}
$$

$Q(z)=z^{n} \overline{P(1 / \bar{z})}$. Using the fact that $B[1]=\lambda_{0}$, we obtain

$$
\begin{aligned}
& \mid(B[P(R z)]+\phi(R, r, \alpha, \beta) B[P(r z)]) \\
& \quad-\mu(1+\phi(R, r, \alpha, \beta)) \lambda_{0} \mid \\
& \leq \mid(B[Q(R z)]+\phi(R, r, \alpha, \beta) B[Q(r z)]) \\
& \quad-\bar{\mu}\left(R^{n}+\varphi(R, r, \alpha, \beta) r^{n}\right) B\left[z^{n}\right] M \mid
\end{aligned}
$$

for all real or complex numbers $\alpha$ and $\beta$ with $|\alpha| \leq 1$, $|\beta| \leq 1, R>r \geq 1$ and $|z| \geq 1$. Now choosing the argument of $\mu$ such that

$$
\begin{aligned}
& \mid(B[Q(R z)]+\phi(R, r, \alpha, \beta) B[Q(r z)]) \\
& -\bar{\mu}\left(R^{n}+\phi(R, r, \alpha, \beta) r^{n}\right) B\left[z^{n}\right] M \mid \\
& =|\mu|\left|R^{n}+\phi(R, r, \alpha, \beta) r^{n}\right|\left|B\left[z^{n}\right]\right| M \\
& -|B[Q(R z)]+\phi(R, r, \alpha, \beta) B[Q(r z)]|
\end{aligned}
$$

which is possible by Theorem 1, we get

$$
\begin{aligned}
|B[P(R z)]+\phi(R, r, \alpha, \beta) B[P(r z)]| \\
-|\mu||1+\phi(R, r, \alpha, \beta)|\left|\lambda_{0}\right| M \\
\leq|\mu|\left|R^{n}+\phi(R, r, \alpha, \beta) r^{n}\right|\left|B\left[z^{n}\right]\right| M \\
\quad-|B[Q(R z)]+\phi(R, r, \alpha, \beta) B[Q(r z)]|
\end{aligned}
$$

for $|\alpha| \leq 1,|\beta| \leq 1, \quad R>r \geq 1$ and $|z| \geq 1$. This implies

$$
\begin{aligned}
& |B[P(R z)]+\phi(R, r, \alpha, \beta) B[P(r z)]| \\
& \quad+|B[Q(R z)]+\phi(R, r, \alpha, \beta) B[Q(r z)]| \\
& \leq|\mu|\left\{\left|R^{n}+\phi(R, r, \alpha, \beta) r^{n}\right|\left|B\left[z^{n}\right]\right|\right. \\
& \left.\quad+\left|\lambda_{0}\right||1+\phi(R, r, \alpha, \beta)|\right\} M,
\end{aligned}
$$

for $|\alpha| \leq 1, \quad|\beta| \leq 1, \quad R>r \geq 1$ and $|z| \geq 1$. Letting $|\mu| \rightarrow 1$, we obtain

$$
\begin{aligned}
|B[P(R z)]+\phi(R, r, \alpha, \beta) B[P(r z)]| \\
\quad+|B[Q(R z)]+\phi(R, r, \alpha, \beta) B[Q(r z)]| \\
\leq\left\{\left|R^{n}+\phi(R, r, \alpha, \beta) r^{n}\right|\left|B\left[z^{n}\right]\right|\right. \\
\left.\quad+\left|\lambda_{0}\right||1+\phi(R, r, \alpha, \beta)|\right\} M,
\end{aligned}
$$

which is inequality (18) and this proves Theorem 2.

Proof of Theorem 3. Lemma 3 and Theorem 2 together yields for all real or complex numbers $\alpha$ and $\beta$ with $|\alpha| \leq 1,|\beta| \leq 1, \quad R>r \geq 1$ and $|z| \geq 1$,

$$
\begin{aligned}
2 & |B[P(R z)]+\phi(R, r, \alpha, \beta) B[P(r z)]| \\
= & |B[P(R z)]+\phi(R, r, \alpha, \beta) B[P(r z)]| \\
& +|B[P(R z)]+\phi(R, r, \alpha, \beta) B[P(r z)]| \\
\leq & |B[P(R z)]+\phi(R, r, \alpha, \beta) B[P(r z)]| \\
& +|B[Q(R z)]+\phi(R, r, \alpha, \beta) B[Q(r z)]| \\
\leq & \left\{\left|R^{n}+\phi(R, r, \alpha, \beta) r^{n}\right|\left|B\left[z^{n}\right]\right|\right. \\
& +|1+\phi(R, r, \alpha, \beta)|\} M,
\end{aligned}
$$

which gives

$$
\begin{aligned}
& |B[P(R z)]+\phi(R, r, \alpha, \beta) B[P(r z)]| \\
& \leq \frac{1}{2}\left\{\left|R^{n}+\phi(R, r, \alpha, \beta) r^{n}\right|\left|B\left[z^{n}\right]\right|\right. \\
& \left.\quad+\left|\lambda_{0}\right||1+\phi(R, r, \alpha, \beta)|\right\} M,
\end{aligned}
$$

which is the Inequality (21) and this completes the proof of Theorem 3.

Proof of Theorem 4. Since $P(z)$ is a self-inversive polynomial of degree $n$, therefore

$$
P(z)=Q(z)=z^{n} \overline{P(1 / \bar{z})}
$$

for all $z \in C$. This implies, in particular, that for all real or complex numbers $\alpha$ and $\beta$ with $|\alpha| \leq 1,|\beta| \leq 1$, $R>r \geq 1$ and $|z| \geq 1$,

$$
\begin{aligned}
& |B[P(R z)]+\phi(R, r, \alpha, \beta) B[P(r z)]| \\
& =\mid B[Q(R z)]+\phi(R, r, \alpha, \beta) B[Q(r z)] .
\end{aligned}
$$

Combining this with Theorem 2, the desired result fol- 
lows immediately. This completes the proof of Theorem 4.

\section{Acknowledgements}

Authors are thankful to the referee for his suggestions.

\section{REFERENCES}

[1] G. V. Milovanovic, D. S. Mitrinovic and Th. M. Rassias, "Topics in Polynomials: Extremal Properties, Inequalities, Zeros," World scientific Publishing Co., Singapore, 1994.

[2] Q. I. Rahman and G. Schmessier, "Analytic Theory of Polynomials," Claredon Press, Oxford,2002.

[3] A. C. Schaffer, "Inequalities of A. Markoff and S. Bernstein for Polynomials and Related Functions," Bulletin of the American Mathematical Society, Vol. 47, No. 2, 1941, pp. 565-579. doi:10.1090/S0002-9904-1941-07510-5

[4] M. Riesz, "Uber Einen Satz des Herrn Serge Bernstein," Acta Mathematica, Vol. 40, 1916, pp. 337-347. doi:10.1007/BF02418550

[5] G. Pólya and G. Szegö, "Aufgaben und Lehrsätze aus der
Analysis," Springer-Verlag, Berlin, 1925.

[6] P. D. Lax, "Proof of a Conjecture of P. Erdös on the Derivative of a Polynomial," Bulletin of the American Mathematical Society, Vol. 50, No. 8, 1944, pp. 509-513. doi:10.1090/S0002-9904-1944-08177-9

[7] N. C. Ankeny and T. J. Rivlin, "On a Theorm of S. Bernstein," Pacific Journal of Mathematics, Vol. 5, 1955, pp. 849-852.

[8] A. Aziz and N. A. Rather, "On an Inequality of S. Bernstein and Gauss-Lucas Theorem," Analytic and Geometriv Inequalities, Kluwer Academic Pub., Dordrecht, 1999, 29-35. doi:10.1007/978-94-011-4577-0 3

[9] Q. I. Rahman, "Functions of Exponential Type," Transactions of the American Mathematical Society, Vol. 135, 1969, pp. 295-309. doi:10.1090/S0002-9947-1969-0232938-X

[10] E. B. Saff and T. Sheil-Small, "Coefficient and Integral Mean Estimates for Algebraic and Trigonometric Polynomials with Restricted Zeros," Journal of the London Mathematical Society, Vol. 9, No. 2, 1975, pp. 16-22

[11] M. Marden, "Geometry of Polynomials," Surveys in Mathematics, No. 3, 1949. 